\title{
Pilot study of ultra-high energy Cosmic rays through their Space - Atmospheric interactions - COSAT
}

\author{
Paula Gina Isar ${ }^{1, a}$ and Doina Nicolae ${ }^{2}$ \\ ${ }^{1}$ Institute of Space Science, Atomistilor Street 409, Magurele 077125, Romania \\ ${ }^{2}$ Institute of Research and Development for Optoelectronics, Atomistilor 409, Magurele 077125, Romania
}

\begin{abstract}
One hundred years after the discovery of cosmic rays, the study of charged ultra-high energy cosmic rays remains a vital activity in fundamental physics. While primary cosmic rays could not be measured directly until it was possible to get the detectors high in the atmosphere using balloons or spacecraft, nowadays very energetic cosmic rays are detected indirectly by ground-based experiments measuring their Extensive Air Showers (EAS) induced Cherenkov and fluorescent light, or radio waves. Moreover, all cosmic ray measurements (performed either from space or ground) rely on accurate understandings of atmospheric phenomena. The concept of the COSAT project is the inter-link between Astroparticle Physics, Remote Sensing and Atmospheric Environment, willing to investigate the energetic cosmic rays physical processes using the atmosphere as a detector in order to identify potential scientific niches in the field of space sciences. A short introduction on the current status and perspectives of the national partnership COSAT project will be given.
\end{abstract}

\section{Introduction}

The COSAT project [1] - "Pilot study of ultra-high energy Cosmic rays through their Space - Atmospheric interactions" - is a two-year (2013-2015) interdisciplinary Romanian national partnership project. The project's goal is to develop an inter-link between astroparticle physics, remote sensing and atmospheric environment, through the ultra-high energy cosmic ray interactions and effects in the Earth's atmosphere, based on their present groundand future space-based measurements. The coordinating organization $(\mathrm{CO})$ is the Institute of Space Science, with project manager Dr. Paula Gina Isar, senior scientist in the Laboratory of High Energy, Astrophysics and Advanced Technology. The partner organization (P1) is the Institute of Research and Development for Optoelectronics, with person in charge Dr. Doina Nicolae, head of Remote Sensing Department.

The Institute of Space Science (ISS, [2]) is a branch of the National Institute of Research and Development for Lasers, Plasma and Radiation Physics, operating under the auspices of the Romanian Ministry for National Education. Its mission is to participate to the world-wide efforts of knowledge and use of the cosmic space for increasing the quality of life on the Earth, and also to have an important contribution for developing and maintaining the Romanian expertise and capabilities in the cosmic space area. ISS has activities on fundamental and applicative research in the following domains: Space Physics (cosmic rays, astrophysics and high energy physics, mathematics, space plasma and magnetometry, cosmology, astroparticle physics), Microgravity and Satellites, Space Technologies (engineering for space researches), Distributed calculus for research, space and ground applications. The research is especially realized in international collaborations with Universities and Institutes within Europe, as well

\footnotetext{
a e-mail: gina.isar@spacescience.ro
}

as through participation in space experiments in the frame of ESA (Planck, EUCLID), ground experiments at CERN, GSI-Darmstadt, IUCN-DUBNA, and at the large international projects ANTARES, KM3Net, and the Pierre Auger Observatory. ISS has collaborated with NASA for the first Romanian experiment on board the International Space Station [3]. Our activities related to the physics assigned to the Pierre Auger cosmicray Observatory involves: high performance resources of CPU and storage for the Auger GRID VO, automatized simulation procedures for coincident AERA (Auger Engineering Radio Array) events, contributions to the Auger surface detector electronics upgrade development for "Auger beyond 2015" and participation to EducationOutreach and Mass-Media activities.

The Institute of Research and Development for Optoelectronics (INOE2000, [4]) is involved in fundamental and applicative research in optoelectronics, analytical chemistry, and high pressure physics. Laser Remote Sensing Department has as long-term mission the strengthening of the research and technological development capacities and building strategic partnerships with excellent research entities worldwide in order to produce and manage information about the environment conforming to global observation strategies. The efforts are focused on two main objectives: 1) development and improvement of investigation methods dedicated to environmental assessment and control; 2) experimental and theoretical research on atmospheric compounds' microphysics, radiative forcing, air quality and climatology, emphasizing short-lived species, in the frame of European and global networks: EARLINET, AERONET, MWRNET, ACTRIS. Our work is devoted to technological improvement of remote sensing instruments and advancing research on air quality and climate change, but we also play a key role in developing remote sensing capacities (instruments and human resources) in our country, by

This is an Open Access article distributed under the terms of the Creative Commons Attribution License 4.0, which permits unrestricted use, distribution, and reproduction in any medium, provided the original work is properly cited. 
setting up the Romanian Lidar Network based on a Romanian Lidar prototype.

\section{Objectives}

The main objectives of the COSAT project [1] are: 1. to investigate the energetic cosmic rays physical processes using the atmosphere as a detector; 2 . to identify potential scientific niches in the field of space science and environment in order to prepare to participate in the European Space Agency - ESA's Optional Programs (e.g. Science, Earth Observation etc.).

Cosmic rays represent a subject of high interest in Astroparticle Physics having various synergies with other sciences. Although their origin [5], sources or production mechanisms are not certain yet, different innovative detection techniques are used to observe them either directly from space or indirectly in different media (like soil, air, water, ice, salt mines etc.). While a primary cosmic ray particle coming from space (i.e. from a supernova explosion or a super-massive black hole) interacts with the Earth's atmosphere, it initiates a cascade of billions of secondary elementary particles forming the so-called extensive air shower.

The world largest observatory of these phenomena, the Pierre Auger Observatory [6] located in Argentina measures cosmic-rays-induced air showers on an area of $3000 \mathrm{~km}$ square through 1660 water-Cherenkov tanks and 27 UV fluorescence telescopes [7], including 160 low frequency radio antennas [8]. Romania joined Auger in 2012 and became a full Auger member in 2014, ISS being one of the four Romanian member research institutes [9].

A potential future space-based cosmic ray observatory is JEM-EUSO [10] to be accomodated on the Japanese Experiment Module (JEM) hosted on the International Space Station. EUSO (Extreme Universe Space Observatory) aims to observe the fluorescence light of cosmic rays air showers from space.

Spacecraft satellites in complement to in-situ measurements may help unravel the cosmic ray mystery. The two cosmic ray observatories, the present Auger and the future JEM-EUSO, are equipped with atmospheric monitoring systems to observe the Earth's atmosphere. Lidar-based atmospheric monitoring at the location of high energy astroparticle detectors are essential to observing cloud caverage and aerosols $[11,12]$. Besides temperature, humidity and pressure, clouds and aerosols affect the shower observation and reconstruction through the e.g. absorbtion and/or reflection of the fluorescence light (i.e. produced by secondary charged particles at the excitation of nitrogen molecules in the air).

The Lidar system uses an optical active remote sensing technique for measuring properties of backscattered light and to find range and other optical information of target layers (the atmospheric constituents). The multiwavelength Raman-depolarization Lidar at INOE is designed for the detection of Mie/Rayleigh backscattered radiation on atmospheric particles and Raman backscattered radiation on atmospheric water vapour and nitrogen. The laser emission wavelengths are $1064 \mathrm{~nm}(90 \mathrm{~mJ})$, $532 \mathrm{~nm}(50 \mathrm{~mJ})$ and $355 \mathrm{~nm}(60 \mathrm{~mJ})$ and the detection

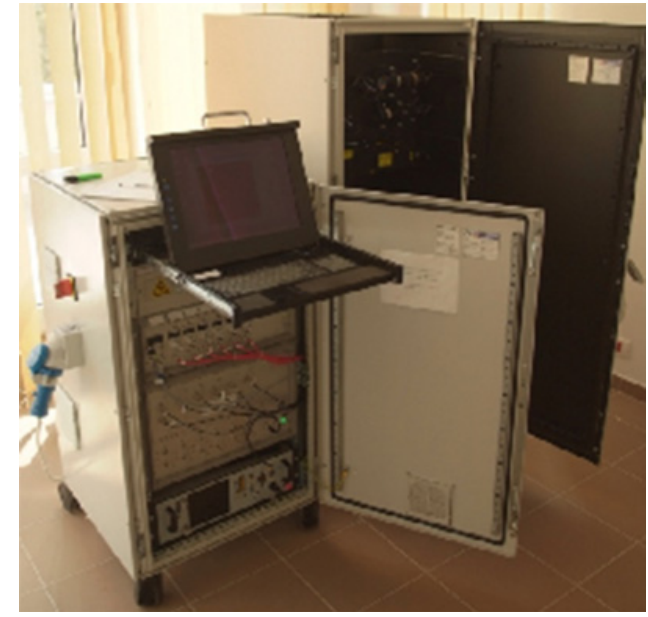

Figure 1. RALI: the multiwavelength Raman depolarization Lidar at RADO, INOE.

channels are 1064, 532 cross, 532 parallel, $355 \mathrm{~nm}$ (elastic wavelengths), and 607, 387 and $408 \mathrm{~nm}$ (water vapour). The dynamic range covers $1-15 \mathrm{~km}$ with a $3.75 \mathrm{~m}$ spatial resolution. By combining all these channels, the system can provide a complete and comprehensive dataset related to the investigated atmospheric structures. The output parameters are the backscatter coefficient, the extinction coefficient, the water vapour mixing ratio (for $407 \mathrm{~nm}$ ) and the particle depolarization ratio (for $532 \mathrm{~nm}$ ). The INOE Lidar system is used in the framework of COSAT for studies related to optical and microphysical properties of aerosols, aerosol typing, cloud formation and for characterization of water vapour in the troposphere. The system is part of the European Lidar Research Network Aerosols, Clouds, and Trace gases Research Infrastructure Network (EARLINET-ACTRIS) and the Romanian Lidar Network (ROLINET) as a reference instrument. RALI (Fig. 1), the multiwavelength Raman depolarization Lidar at the INOE-RADO - Romanian Atmospheric research 3D Observatory measures profiles of aerosols, clouds and water vapor, and makes correlative measurements during overpasses of CALIPSO (The Cloud-Aerosol Lidar and Infrared pathfinder Sattelite Observation [13]), in the framework of EARLINET's program.

\subsection{Contributions and results}

The project's contribution to the goal of the funding agency are the following:

1. Identify research niches at national, European and international level;

2. Promote the research field in education and mass media for public;

3. Continuous inter-disciplinary training; attracting and educating young students;

4. Produce qualitative achievements - increase the quality of scientific research;

5. Organize scientific outreach events for education, society and industry. 


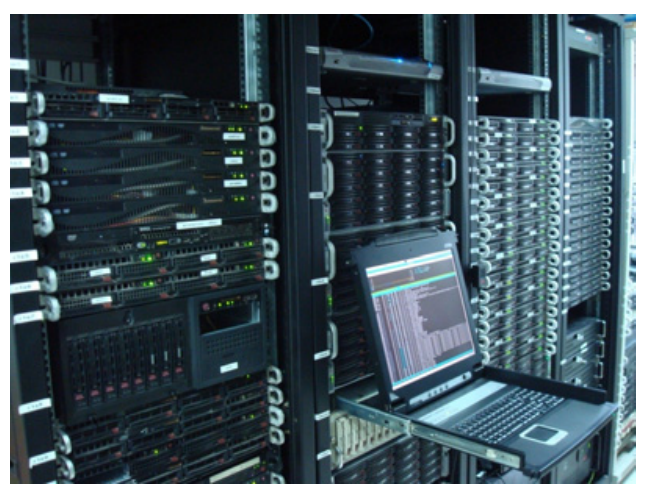

Figure 2. GRID Data Center infrastructre at the ISS. ISS is contributing with $90 \mathrm{CPU}$ and $100 \mathrm{~TB}$ storage for the Auger GRID VO.

The project's results obtained so far are the following:

1. Software and hardware HPC infrastructure (see Fig. 2).

2. Atmospheric measurements by INOE - on going.

3. Seminars, reportages, outreach events - permanent updated activity on the project web page [1].

The project results are envisaged to come in support to ESA - European Space Agency Science Program (Cosmic Vision, EUSO Mission), space and atmospheric weather, Earth Observation Program Copernicus (Atmosphere services and Sentinels 4 and 5).

Dissemination activities of the project are continuously performed very broadly, from outreach to education and mass media. They can be followed at the project web page [1]. Project members of both participating institutions $(\mathrm{CO}, \mathrm{P} 1)$ have contributions at both national and international workshops, conferences and summer schools, local school visits and mass media.

The author PGI acknowledges the fruitful collaboration between the project's partner research institutes.
This work was supported by a grant of the Minister of National Education, Programme for research - Space Technology and Advanced Research - STAR, project number 83/2013.

\section{References}

[1] www.spacescience.ro/projects/cosat

[2] www.spacescience.ro

[3] Gomoiu I, Chatzitheodoridis E, Vadrucci S, Walther I., The effect of spaceflight on growth of Ulocladium chartarum colonies on the International Space Station. PLOS ONE, DOI: 10.1371/journal.pone.0062130 (2013)

[4] www.inoe.ro

[5] Abreu P. et al., Pierre Auger Collaboration, Constraints on the Origin of Cosmic Rays above $1018 \mathrm{eV}$ from Large-scale Anisotropy Searches in Data of the Pierre Auger Observatory, The Astrophysical Journal Letters, 756, p. 8 (2013)

[6] www .auger.org

[7] Abreu P. et al, Pierre Auger Collaboration, The exposure of the hybrid detector of the Pierre Auger Observatory, Astroparticle Physics, 34, Issue 6, p. 368-381 (2011)

[8] Aab A., et al., Pierre Auger Collaboration, Probing the radio emission from air showers with polarization measurements, Physical Review D, 89, Issue 5, id.052002 (2014)

[9] www2.spacescience.ro/?s=auger

[10] Adams J.H. et al., JEM-EUSO Collaboration, An evaluation of the exposure in nadir observation of the JEM-EUSO mission, Astroparticle Physics 44, p. 76-90 (2013)

[11] Abreu P. et al., Pierre Auger Collaboration, Identifying clouds over the Pierre Auger Observatory using infrared satellite data, Astroparticle Physics 50, p. 92-101 (2013)

[12] The Pierre Auger Collaboration, The rapid atmospheric monitoring system of the Pierre Auger Observatory, Journal of Instrumentation, 7, p. 9001 (2012)

[13] http://www-calipso.larc.nasa.gov 